\title{
Surface Electromyography to Identify Laryngeal Tension in Selective Mutism: Could this be the Missing Link?
}

\author{
Cesar E Ruiz* and Evelyn R Klein* \\ La Salle University, Department of Communication Sciences and Disorders, Philadelphia, PA
}

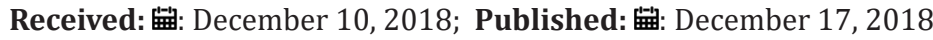

*Corresponding author: Cesar E Ruiz and Evelyn R Klein. La Salle University, Department of Communication Sciences and Disorders, Philadelphia

\begin{abstract}
Selective mutism (SM) is an anxiety based disorder that impedes the ability to speak in varied situations. The purpose of this paper is to share a study providing information using surface electromyography to measure laryngeal tension for voice initiation in 33 children with SM. Results indicated that elevated laryngeal tension was present when children with SM were asked to produce vowel sounds and speech. Requests for speech at the beginning of the evaluation process produced significantly more tension when compared to measures obtained after children became more familiar with the setting and evaluators. The findings provide a better understanding of the physiological consequences of anxiety on voice production at the level of the larynx in children with SM.
\end{abstract}

Keywords: Selective Mutism; Surface Electromyography; Laryngeal tension; Vocal Control

Abbreviations: SM: Selective Mutism; sEMG: Surface Electromyography; ACC: Anterior Cingulate Cortex; PAG: Periaqueductal Grey; GMV: Gyrus Gray Matter Volumes

\section{Introduction}

In order to speak, an individual needs to have the ability to initiate phonation (voice), as well as to turn it 'on' and 'off' as needed. Such ability depends on well controlled laryngeal muscle activity paired with coordination with respiratory and articulatory (oral) systems. Normal vocalization requires that air within the lungs flows outward in a steady manner while the vocal folds approximate to initiate vibration (voice). Then the voice travels outwards toward the articulators (tongue, teeth, lips, palate, etc.) where it is shaped into speech sounds to form words. Although individuals may be able to automatically or reflexively activate each system independently, unless a voluntary control of those systems exists, speech production is not possible [1]. Selective Mutism (SM) is an anxiety disorder in which the individual experiences failure to speak in specific social situations where there is an expectation to speak such as at school despite being able to speak in situations where anxiety is low, such as at home with family. Lack of speech is not due to a lack of knowledge [2]. Rather, physiological sensations of anxiety appear to impact the person's ability to speak.

The purpose of this short communication is to share a systematic assessment procedure that focuses on vocal initiation and control to provide a better understanding of the physiological consequences of anxiety on voice production at the level of the larynx. Prevalence estimates of SM range from $0.03 \%$ to $2 \%$. Onset is often between 3 and 6 years. Symptoms are typically present in the early school age years [3]. The ability to vocalize can be divided into involuntary (crying, coughing, etc.) and voluntary (speech production) functions. It is important to understand such a distinctive division because the neurological pathways associated with each behavior originate in different parts of the nervous system.

Involuntary voicing, also described as innate vocalizations, are controlled at the level of the sensory and motor nuclei of the lower brain stem and spinal cord. These areas are responsible for basic interactions and coordination of the laryngeal, respiratory and articulatory systems [1,4]. Voluntary vocalizations, as those needed for speech production, are controlled by communication between the Anterior Cingulate Cortex (ACC) and the Periaqueductal Grey (PAG), which are associated with purposeful voice initiation and its emotional implications [1,4]. The ACC has been described as a specialization area responsible for emotional self-control, problem solving, and adaptive responses to changing conditions [5]. Our alternative physiological explanation for the inability to speak by those with SM is based on the understanding of intricate 
interactions between the ACC and the PAG in translating intentions into actions. In patients with anxiety disorders, reductions in the right anterior cingulate cortex and the left inferior frontal gyrus gray matter volumes (GMVs), were identified [6].

Further research indicates that anxiety compromises the function of the ACC and that socially anxious individuals showed less ACC activation than control subjects. This compromised cognitive control and emotion regulation, likely reduced the ability to purposefully initiate vocalizations for voluntary speech production [7-9]. Social anxiety and physiological tension in the vocal mechanism often make it difficult and uncomfortable for those with SM to vocalize [10]. We speculate that the adult in this previous study by Ruiz and Klein, who was selectively mute as a child from kindergarten through 12th grade, experienced increased difficulty initiating voice due to sensations of tension in his vocal system. He clearly described his inability to get his voice started along with feelings of someone grabbing his throat, despite his desire to speak. His recounts prompted the incorporation of a systematic vocal assessment using Surface Laryngeal Electromyography (sEMG) with children who were diagnosed with SM by a licensed psychologist or specialized physician.

\section{Material and Methods}

Participants included 33 children (14 females, 19 males) ranging from 2.42 years to 13.75 years (Mean $=7.20$ years, SD $=2.39$ years) who were evaluated at an outpatient university communication disorders clinic in a major metropolitan city in the Northeast part of the U.S. Children came from a variety of ethnic backgrounds (Caucasian $=54.5 \%$, Asian $=18.2 \%$, Hispanic $=18.2 \%$, Bi-racial $=6.1 \%$, and Eastern European=3.0\%). There were no sensory deficits for hearing or vision and none of the children had intellectual disability. All Pulse/Ox readings were within normal limits both at the beginning (pre) of the testing time (Pulse Mean =96.07, Pulse $\mathrm{SD}=18.09$, Oxygen Mean = 96.77, Oxygen $\mathrm{SD}=2.38$ ) and again at the end (post) of testing (Pulse Mean $=94.10$, Pulse $\mathrm{SD}=14.57$, Oxygen Mean $=97.00$, Oxygen $\mathrm{SD}=2.23$ ) which took approximately three hours in total and included speech articulation, fluency, language assessment, hearing screening, and the voice evaluation. Two children would not permit sensors to be placed on their necks so voice data was collected from 31 children. The Trigno Mini Wireless Surface Electromyography (sEMG) system MAN-0201-1 by Delsys, Inc., calibrated for fine motor studies with difficult to isolate muscles, was used to obtain readings of laryngeal tension. A sensor ( $25 \times 12 \times 7 \mathrm{~mm})$ was placed on the side of the larynx (thyroid cartilage) to measure changes in muscle tension (thyrohyoid) when the child was (1) seated at rest, (2) asked to imitate a vowel sound, and (3) asked to say their name, (i.e., speaking on command) [11].

Tension measures excluded peaks during swallowing. Measures of tension were based on readings prior to phonation that declined once speech was produced. During this testing, blood oxygenation and heart rate were also measured with the Burdick Oxy100 (LR 78469) Pulse/Ox device. Children tolerated the testing without difficulty. As part of this university IRB-approved study, parents consented to the testing process with their children. The primary question we intended to answer was: Do sEMG levels measuring laryngeal tension change beyond 18 microvolts for children diagnosed with SM? We also questioned if there would be a difference in laryngeal tension for the three testing conditions (at rest with 1- no vocal requests, 2- vowel imitation, 3- speech with a vocal request to say their name) at the beginning (pre) and end (post) of the comprehensive speech-language evaluation process (before and after children become more accustomed to and familiar with the setting and people). Children entered the testing room accompanied by parents. They were informed that there would be no sensations from the sensor and that it was only used to gather information. The sEMG sensor was placed on the right side of the thyroid cartilage and remained there throughout the voice testing. Pulse-Ox was placed on the children's right index finger at the start of the testing to collect readings. Readings at rest were taken. Next, children were shown an App that changes images when voice is produced. The evaluator said a vowel sound and asked the children to imitate it. The same process was done when the children were asked to say their names.

\section{Results}

The results of sEMG measures indicated that children with SM displayed significantly greater laryngeal tension that increased with speech demands. The greatest tension was identified when children were asked to say their names at the beginning (pre) of the testing session. Slightly less laryngeal tension was recorded when asked to repeat the vowel sound of /i/ as in "eagle" and the least tension was recorded when seated at rest (not asked to say anything). Reductions in the number of children who took part in the post test phase was due to fatigue and scheduling conflicts. A chi-square test of independence was calculated comparing age groups of children at pre and post evaluation times. No significant relationship was found $\left(\mathrm{X}^{2}=6.00, \mathrm{df}=4, \mathrm{p}>.05\right.$ ) (Table 1). A $3 \times 2$ (sEMG task by pre-post evaluation) repeated-measures ANOVA was calculated. A significant interaction effect was found $F(1.46,24.75)$ $=6.61, p=.009$. The greatest laryngeal tension was identified at the beginning of the testing session when children were asked to say their names.

Table 1: Repeated measures ANOVA means and standard deviations with effect sizes for time (pre-post).

\begin{tabular}{|c|c|c|c|}
\hline sEMG Task & $\begin{array}{c}\text { Pre-Evaluation } \\
\boldsymbol{M}(\boldsymbol{S D})\end{array}$ & $\begin{array}{c}\text { Post } \\
\text { Evaluation } \boldsymbol{M} \\
\text { (SD) }\end{array}$ & $\begin{array}{c}\text { Effect Size } \\
\text { Cohen's d }\end{array}$ \\
\hline $\begin{array}{c}\text { sEMG at rest } \\
\text { (level } 1)\end{array}$ & $\begin{array}{c}15.67(6.52) \\
n=31\end{array}$ & $\begin{array}{c}13.30(5.04) \\
n=23\end{array}$ & .41 (small) \\
\hline $\begin{array}{c}\text { sEMG asked to } \\
\text { repeat vowel } \\
\text { (level 2) }\end{array}$ & $\begin{array}{c}24.74(11.12) \\
\mathrm{n}=27\end{array}$ & $\begin{array}{c}21.05(12.26) \\
\mathrm{n}=20\end{array}$ & .32 (small) \\
\hline $\begin{array}{c}\text { sEMG asked } \\
\text { to say name } \\
\text { (level3) }\end{array}$ & $\begin{array}{c}35.11(20.21) \\
n=28\end{array}$ & $\begin{array}{c}21.18(13.97) \\
n=22\end{array}$ & .80 (large) \\
\hline
\end{tabular}

There was a significant main effect for sEMG task, $F(1.39$, $23.67)=18.22, p<.001$. Pairwise comparisons for the main effect of the sEMG task, using a Bonferroni adjustment, indicated significant differences between levels 3 and 2 and also levels 3 and 1 (greater laryngeal tension when children were asked to say their names compared to when at rest or when asked to repeat a vowel sound). 
There was also a significant difference ( $p=.023)$ between levels 2 and 1 (greater laryngeal tension when asked to say a vowel sound than when at rest). There was also a significant main effect for pre-post evaluation times, $F(1,17)=5.80, p<.028$. Pairwise comparisons for the main effect of time (pre and post evaluation) indicated greater laryngeal tension at pre evaluation time than at post evaluation time across all tasks with more demanding speech requests producing the greatest laryngeal tension for children with SM (Figure 1).

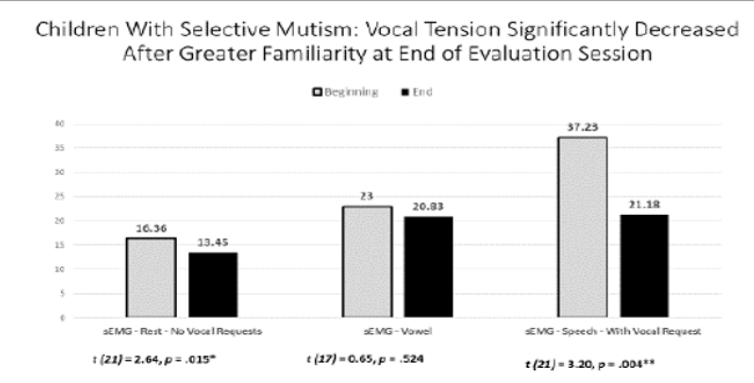

Figure 1: Paired t-test results of laryngeal tension levels of children with selective mutism at beginning (pre) and end (post) evaluation times.

\section{Conclusion}

Children with SM do not speak in settings where they feel uncomfortable. This usually includes places where people are less familiar and where there are performance expectations to speak such as at school. The question is, "Why is speech output affected in this population of children with anxiety?" There were many children in the sample we evaluated that would not speak to use the bathroom in school, to get lunch when hungry, or respond to get passing grades when required to read aloud. SM is a debilitating disorder that impacts the entire family as parents struggle to make sense of why their children do not speak in some situations. Speech becomes the focus for these families with increased frustration. After interviewing adults who have recovered from SM, it became evident that they wanted to talk as children but were unable to do so when anxious. Anxiety can affect individuals in varied ways and so we speculate that anxiety may be preventing the children's ability to make their vocal mechanism work adequately to speak. Anxiety compromises the function of the anterior cingulate cortex (ACC). This has been documented in socially anxious individuals. Compromised cognitive control and emotion regulation due to anxiety may reduce the ability to purposefully initiate vocalizations for voluntary speech. This study is the first to attempt to identify a breakdown in the vocal mechanism impacting the ability to initiate voice. Voice for speech purposes is a voluntary act. We speculate that the laryngeal system is not effectively engaging the vocal cords to adduct and abduct properly to vibrate for speech. In this study we found that children had greater laryngeal tension at the level of the thyrohyoid muscle (measured by sEMG) when asked to say their names than they did for repeating a vowel sound and they had significantly more laryngeal tension than when measures were taken at rest (without any requests for vocalization). With greater familiarity over the time children with SM spent in the evaluation session, levels of laryngeal tension decreased to a statistically significant degree. Professionals working with individuals who have a diagnosis of SM are encouraged to consider that children may not be able to speak, even if they want to and that their lack of talking is not due to defiance or an oppositional disposition. These findings are important to the future study and treatment of this complex disorder.

\section{References}

1. Belyk M, Brown S (2017) The origins of the vocal brain in humans. Neuroscience \& Biobehavioral Reviews 77: 177-193.

2. (2013) Diagnostic and statistical manual of mental disorders ( $5^{\text {th }} \mathrm{edn}$ ).

3. Rhoads JC, Donnelly CL (2012) Anxiety disorders in childhood and adolescence. Clinical Child Psychiatry 3: 215-242.

4. Simonyan K, Horwitz B (2011) Laryngeal motor cortex and control of speech in humans. The Neuroscientist 17(2): 197-208.

5. Allman JM, Hakeem A, Erwin JM, Nimchinsky E, Hof P (2006) The anterior cingulate cortex: Evolution of an interface between emotion and cognition. Annals of the New York Academy of Sciences 935(1): 107-117.

6. Shang J, Fu Y, Ren Z, Zhang T, Du M, et al. (2014) The common traits of the ACC and PFC in anxiety disorders in the DSM-5: Meta-analysis of voxelbased morphometry studies. PLoS ONE 9(3): e93432.

7. Klumpp H, Angstadt M, Phan KL (2012) Insula reactivity and connectivity to anterior cingulate cortex when processing threat in generalized social anxiety disorder. Biological Psychology 89(1): 273-276.

8. Etkin A, Egner T, Kalisch R (2011) Emotional processing in anterior cingulate and medial prefrontal cortex. Trends in Cognitive Sciences 15(2): 85-93.

9. Etkin A, Prater KE, Hoeft F, Menon V, Schatzberg AF (2010) Failure of anterior cingulate activation and connectivity with the amygdala during implicit regulation of emtional processing in generalized anxiety disorder. American Journal of Psychiatry 167(5): 545-554.

10. Ruiz C, Klein ER (2014) The effects of anxiety on voice production: A retrospective case report of selective mutism. Pennsylvania SpeechLanguage-Hearing Association Journal 4: 19-26.

11. In Stepp, CD (2012) Surface electromyography for speech and swallowing systems: Measurement, analysis, and interpretation. Journal of Speech, Language, and Hearing Research 55(4): 1232-1246. 


\section{ISSN: 2574-1241}

DOI: $10.26717 / B J S T R .2018 .12 .002222$

Cesar E Ruiz, Evelyn R Klein. Biomed J Sci \& Tech Res

(C) (i) This work is licensed under Creative

Submission Link: https://biomedres.us/submit-manuscript.php

$\begin{array}{ll}\text { BIOMEDICAL } & \text { Assets of Publishing with us } \\ \text { RESEARCHES } & \text { - Global archiving of articles } \\ & \text { - Immediate, unrestricted online access } \\ & \text { - Rigorous Peer Review Process } \\ \end{array}$

